\title{
PSICOLOGIA E PRÁTICAS PSICOSSOCIAIS: NARRATIVAS E CONCEPÇÕES DE PSICÓlOGOS DE CENTROS DE ATENÇÃO PSICOSSOCIAL
}

\author{
Thaís Thomé Seni da Silva e Oliveira \\ Departamento de Saúde Mental de Trieste
}

\author{
Regina Helena Lima Caldana \\ Universidade de São Paulo
}

\begin{abstract}
Resumo
A atenção psicossocial, modelo de cuidado em saúde mental vigente no Brasil, preconiza o trabalho interdisciplinar, intersetorial e territorial. Este trabalho tem como objetivo apresentar concepções de psicólogos dos Centros de Atenção Psicossocial de uma cidade paranaense sobre as práticas psicossociais desenvolvidas em seu cotidiano. Foram realizadas entrevistas individuais semiestruturadas e encontros grupais, utilizando-se a técnica de Grupo Operativo de Pichón-Rivière. As entrevistas e os grupos foram gravados, transcritos na íntegra e analisados qualitativamente. Os resultados apontam a dicotomia entre o fazer clínico e o fazer psicossocial e a identidade profissional dos participantes atrelada ao modelo clínico tradicional da psicologia. Algumas práticas psicossociais estão gradualmente sendo reconhecidas pelos profissionais como práticas legítimas da Psicologia, e poderiam ser consideradas clínica ampliada dentro da área. Conclui-se que para a efetivação de práticas psicossociais, é imprescindível a transformação nos cursos de formação e a criação de estratégias de educação permanente para os profissionais.
\end{abstract}

Palavras-chave: centros comunitários de saúde mental; intervenção psicossocial; atendimento psicológico.

\section{PSYCHOLOGY AND PSYCHOSOCIAL PRACTICES: NARRATIVES AND CONCEPTIONS OF PSYCHOLOGISTS FROM THE PSYCHOSOCIAL CARE CENTERS}

\begin{abstract}
The psychosocial care, current care model in mental health in Brazil, emphasizes interdisciplinary, inter-sectoral and territorial actions. This paper aims to present conceptions of psychologists from the Centers for Psychosocial Care of a city on Parana state, about the psychosocial practices developed in their daily actions. Semistructured individual interviews and group meetings were conducted, using the technique of Operating Group of Pichón-Rivière. The interviews and groups were recorded, transcribed and qualitatively analyzed. Results point the dichotomy between clinical and psychosocial practices in psychology and the professional identity of the participants tied to traditional clinical psychology model. Some psychosocial practices are gradually being recognized by professionals as legitimate practice of psychology, and could be considered amplified clinic in psychology. It is concluded that for the effectiveness of psychosocial practices, it is essential to improve graduation courses and permanent education strategies for mental health professionals.

Keywords: community mental health centers; psychosocial intervention, psychological practices.
\end{abstract}




\title{
PSICOLOGIA Y PRÁTICAS PSICOSOCIALES: NARRATIVAS Y CONCEPCIONES DE PSICÓLOGOS DE CENTROS DE ATENCIÓN PSICOSOCIAL
}

\begin{abstract}
Resumen
La atención psicosocial, modelo de cuidado en salud mental del Brasil, preconiza el trabajo interdisciplinar, intersocial y territorial. Este trabajo tiene como objetivo presentar concepciones de psicólogos de los Centros de Atención Psicosocial de una ciudad paranaense sobre las prácticas psicosociales desarrolladas en su cotidiano. Se realizaron entrevistas individuales semiestructuradas y encuentros grupales, utilizando la técnica de Grupo Operativo de Pichòn-Rivière, que fueron grabados, transcritos y analizados cualitativamente. Los resultados señalan la dicotomía entre hacer clínico y hacer psicosocial y la identidad profesional de los participantes nel modelo clínico tradicional de la psicología. Algunas prácticas psicosociales están gradualmente siendo reconocidas por los profesionales como prácticas legítimas de la Psicología, y podrían ser consideradas clínica ampliada dentro del área. Se concluye que para que se vuelvan efectivas las prácticas psicosociales, es imprescindible la transformación de los cursos de formación y la creación de estrategias de educación permanente para los profesionales.
\end{abstract}

Palabras clave: centros comunitários de salud mental, intervención psicosocial, atendimento psicológico.

\section{INTRODUÇÃO}

O modelo de assistência em saúde mental vigente no Brasil, fruto do movimento da luta antimanicomial e da reforma psiquiátrica, é atualmente denominado Atenção Psicossocial e inspirado principalmente na Psiquiatria Democrática Italiana (Amarante, 2011). Foi incorporado como política pública de assistência em saúde mental no Brasil, especialmente após a publicação da lei 10.216 em 2001, e consolidado em uma rede de serviços de saúde mental pela portaria publicada dez anos depois (Ministério da Saúde, 2011).

A Atenção Psicossocial constitui um modelo e uma estratégia de cuidado em saúde mental de base comunitária, e não constitui apenas uma rede de serviços que se propõe a substituir o manicômio, mas representa outro paradigma de cuidado: o paradigma psicossocial, que busca superar o paradigma biomédico e asilar (Costa-Rosa, 2000). No cenário internacional, fala-se da transição em termos semelhantes, partindo do "biological-medical model" para o modelo de "psychosocial approaches" (Mezzina, 2005).

Em relação à mudança paradigmática, Mezzina (2005) aponta uma "ruptura revolucionária", que vai do modelo médico-biológico de tratar a doença e das instituições totais para a organização de serviços humanos, inseridos nas comunidades e que consideram o paciente como sujeito e não como objeto. Isso implica, segundo o autor, na mudança de concepção sobre o que seja tratar/cuidar, sendo a primeira centrada no controle de sintomas e comportamentos, da fragmentação do usuário e da distância entre este e o profissional, e a segunda voltada para a compreensão do sofrimento da pessoa real, seu contexto de vida e suas necessidades concretas, incluindo aquelas psicológicas e subjetivas. 
A saúde mental passa a situar-se no campo da saúde coletiva, sendo o processo saúde-doença resultante de processos biopsicossociais complexos, que demandam uma abordagem interdisciplinar e intersetorial, com ações inseridas em uma rede de serviços e dispositivos de atenção e de cuidado inseridos no território, sendo um deles o Centro de Atenção Psicossocial - CAPS.

Uma vez que a comunidade é o novo cenário para o cuidado em saúde mental, o desafio se torna construir práticas que, de fato, sejam substitutivas às instituições totais. Dessa maneira, o paradigma psicossocial é desafiado pelas práticas que se constroem cotidianamente em cada serviço. Para que serviços comunitários realmente inovadores existam, é necessário evitar os riscos de se produzir instituições fragmentadas e manicômios difusos (Mezzina, 2005).

Concordamos com Yasui e Costa-Rosa (2008) ao dizerem que os profissionais das equipes de saúde mental cotidianamente têm a tarefa de expandir e consolidar essa mudança. Seus principais instrumentos são a sua formação e o desenvolvimento profissional permanente, que devem permitir a redefinição e reorganização de seu processo de trabalho, a articulação das alianças entre os diferentes setores da sociedade; viabilizando a criação e expansão concreta de uma rede de atenção e cuidado de base territorial e pautada nos princípios de integralidade e participação popular.

\section{Práticas em construção: Psicologia e Atenção Psicossocial}

Para compreender as práticas em psicologia nesse contexto, é importante entender o processo histórico da atuação deste profissional no Brasil. Desde a regulamentação da profissão no país, a atuação do psicólogo passou por um processo de transformação bastante importante, resultante de uma diversidade de fatores como: a história da psicologia como ciência no mundo e no Brasil, o contexto histórico e social da atuação profissional e dos cursos de formação, a configuração socioeconômica e política do país, os paradigmas de conhecimento, de educação e de saúde, entre outros.

Pereira e Pereira Neto (2003) destacam que a formação profissional em psicologia, como curso de nível superior, teve início no ano de 1957 com a criação dos dois primeiros cursos de psicologia no país. A regulamentação da profissão de psicólogo data de 27 de agosto de 1962, pela Lei 4.119. Em 1971 foram criados os Conselhos Federal e Regionais de Psicologia, e em 1975 o Conselho Federal de Psicologia publicou o primeiro Código de Ética dos psicólogos, finalizando o processo de profissionalização da área no país.

No que se refere às áreas de atuação do psicólogo, segundo Pereira e Pereira Neto (2003), entre os anos de 1970 e 1980 houve um aumento da demanda por serviços de psicologia nos grandes centros, predominantemente nas classes média e alta, com atuação em três grandes áreas: educação, trabalho e clínica, tendo esta última sempre despertado maior interesse dos profissionais. 
Destaca-se que, nesse período, o Brasil vivia o momento histórico da repressão e da ditadura militar, o que contribuiu para o direcionamento da psicologia e de outras áreas profissionais de determinada maneira.

Predominava na escolha dos psicólogos a área clinica no contexto privado com atendimentos individuais em psicoterapia, tendo a profissão de psicólogo se estruturado à luz do modelo médico/ profissional liberal. Assim, a prática da psicologia no Brasil esteve e está ainda, em grande medida, caracterizada pela predominância do modelo clínico tradicional (Conselho Federal de Psicologia, 2005; Dimenstein, 2000; Dimenstein e Macedo, 2012; Figueiredo \& Rodrigues, 2004; Yamamoto, 2012). A escolha preferencial dos profissionais psicólogos pela área clínica coloca em evidencia este outro fator de extrema relevância: a característica dos cursos de formação em psicologia no Brasil, com predomínio do ensino da área clinica em seus moldes tradicionais, como área privilegiada para a atuação do psicólogo.

\section{Ampliando espaços de atuação para a psicologia.}

A década de 1990 marca a expansão dos espaços de atuação dos psicólogos. Segundo Pereira e Pereira Neto (2003), a preferência pela área clinica se mantém, seguida pelas áreas da saúde e organizacional. Os estudos de Yamamoto (2012) confirmam a predominância dessas áreas de atuação, sendo que 53\% dos psicólogos pesquisados trabalhavam em clínica, 27,9\% na saúde, $25,1 \%$ na área do trabalho e organizações e 9,8\% na área educacional. Porém, o autor sugere mudanças em relação às modalidades de inserção do profissional no país, sendo que apenas um quinto destes profissionais autônomos atuava exclusivamente nessa condição. Ainda, $40 \%$ dos psicólogos participantes da pesquisa trabalhavam no setor público, $35 \%$ no setor privado e $25 \%$ no terceiro setor.

Os dados acima apontam uma expansão da atuação profissional em psicologia para além da clínica privada. Na área da saúde pública, a entrada do psicólogo se intensificou no final da década de 1980, sendo a psicologia a categoria profissional que teve o maior número de profissionais contratados para compor equipes multiprofissionais nas últimas décadas (Yamamoto, 2012).

Porém, tal ampliação não correspondeu a uma transformação das práticas psicológicas. Estudos indicam a permanência de estratégias tradicionais de intervenção como a avaliação psicológica, o psicodiagnóstico e a psicoterapia individual (Yasui, 2010). Assim, nos deparamos efetivamente com a predominância do modelo clinico tradicional (Yasui, 2010; Yamamoto, 2012) e com inúmeras criticas a este por não apresentar resolutividade, despreocupandose com problemas sociais e trazendo forte conteúdo ideológico individualista (Dimenstein \& Macedo, 2012).

No que se refere à atuação na saúde pública, é imprescindível construir novos olhares e fazeres em Psicologia, que ultrapassem o modelo clínico 
tradicional (Dimenstein, 2000, 2006; Ferreira Neto, 2008; Figueiredo \& Rodrigues, 2004; Spink, 2003; Yasui, 2010).

Concordamos com Dimenstein (2006) ao apontar a necessidade de superar a distância entre as demandas da população usuária dos serviços públicos de saúde e o tipo de formação em psicologia. A produção do cuidado em saúde implica em escolhas teóricas e metodológicas que devem possibilitar a concretização de um fazer comprometido com os processos relacionais e com a construção de sujeitos sociais ativos na produção de saúde, seja individual, seja coletivamente.

Para isso preconiza-se a criação de estratégias que permitam ao psicólogo agir dentro de um modelo centrado no usuário, na geração de autonomia e protagonismo social, proposta que se afina de perto com os princípios do paradigma psicossocial.

O desafio da formação profissional vai além da ampliação de disciplinas e conteúdos relacionados ao SUS e à atenção psicossocial, bem como da instrumentalização do profissional com tecnologias sofisticadas, devendo oferecer uma formação que proporcione uma visão ampla e análises conjunturais a respeito das necessidades sociais e de saúde da população, a escuta e a intervenção sobre processos psicológicos e psicossociais mobilizados pelas condições de vida e projetos de futuro da população, além da capacidade de articulação com as redes de serviços para operar práticas de cuidado mais integradas.

Para finalizar este tópico, é importante ressaltar que a construção de instrumentos e tecnologias de cuidado para além do tradicional e do hegemônico, ou seja, o movimento de construção de práticas assistenciais coerentes com os pressupostos do paradigma psicossocial (Amarante, 2011; Yasui \& Costa-Rosa, 2008) está presente na área da psicologia, como também em todas as áreas da saúde no trabalho interdisciplinar que a proposta envolve. Em termos da formação, existem atualmente diversas ações e projetos de ensino, pesquisa e extensão das universidades em parceria com órgãos governamentais como o PET-Saúde e outros programas. Porém, tais ações precisam ser ampliadas na formação e alcançar também as estratégias de educação permanente para os profissionais, para que tenham maior alcance nas práticas cotidianas.

O presente trabalho constitui um recorte de uma pesquisa de doutorado, e visa contribuir com reflexões críticas acerca das práticas psicossociais de psicólogos em Centros de Atenção Psicossocial e dos desafios da formação em psicologia.

\section{MÉTODO}

Este estudo se insere nas abordagens qualitativas de pesquisa, que se articulam ao Paradigma Interpretativo, preconizando uma abordagem 
compreensivista ou interpretativista do objeto de estudo; nele se prioriza a compreensão da perspectiva dos envolvidos no fenômeno e interpretações plausíveis são preferíveis a explicações causais (Bogdan \& Biklen, 1994; Silva, 1998).

\section{Participantes}

Os dados do presente estudo foram coletados nos Centros de Atenção Psicossocial de uma cidade de aproximadamente 500 mil habitantes situada na região norte do estado do Paraná. Sendo eles:

- Centro de Atenção Psicossocial - CAPS III;

- Centro de Atenção Psicossocial - CAPS Infantil;

- Centro de Atenção Psicossocial - álcool e drogas - CAPS AD.

Participaram desta pesquisa 12 psicólogos que integravam as equipes dos três CAPS referidos. Na época da realização da pesquisa as equipes somavam 15 profissionais, distribuídos entre estas. Os três profissionais que não participaram do estudo alegaram incompatibilidade com os horários da pesquisa.

$\mathrm{Na}$ divisão por tipo de serviço, participaram das entrevistas e dos encontros grupais quatro dos sete psicólogos do CAPS III, todos os quatro psicólogos do CAPS i e todos os quatro psicólogos do CAPS AD. Os participantes eram mulheres em sua maioria, com idade entre 30 e 48 anos, com tempo de graduado em Psicologia entre cinco e 25 anos e tempo de trabalho nos serviços pesquisados entre 3 meses e 12 anos.

Destaca-se que os nomes dos entrevistados presentes nas falas apresentadas são fictícios visando preservar a identidade dos mesmos.

\section{Instrumentos}

O trabalho de pesquisa se deu por meio da utilização de dois instrumentos para a obtenção dos dados:

1 - Entrevistas semi-estruturadas: entrevistas individuais por meio de um roteiro de entrevista semi-estruturado a partir de tópicos norteadores e complementares, com duplo objetivo: a investigação das concepções individuais dos participantes sobre suas praticas profissionais, e o levantamento de temas para a etapa posterior.

2 - Realização de grupos com os participantes. O segundo momento da pesquisa consistiu na realização de encontros grupais, utilizando-se a técnica do Grupo Operativo de Enrique Pichón-Rivière (2000a, 2000b).

\section{Procedimentos}

O inicio do procedimento de obtenção dos dados da pesquisa se deu pelo contato com a gerência de saúde mental do município, e, posteriormente pelo contato com as equipes de cada serviço CAPS para apresentação do projeto de pesquisa e convite para a participação. 
A Secretaria de Saúde do Estado do Paraná compõe sua rede através de 22 Regionais de Saúde. A cidade em questão, que tem população atual aproximada de 500 mil habitantes, é a sede de uma das regionais de saúde, da qual fazem parte 20 municípios.

As entrevistas individuais com os profissionais foram gravadas $e$ transcritas na íntegra, tiveram duração média de uma hora e foram realizadas no próprio local de trabalho dos entrevistados. De modo geral, os participantes se mostraram bastante receptivos no momento da entrevista, interagindo bastante com a pesquisadora e relatando de maneira detalhada suas vivências.

Finalizada a etapa das entrevistas individuais, foram realizados seis encontros grupais, de frequência semanal e duração de uma hora e meia em média, todos gravados e transcritos na íntegra. Os encontros grupais ocorreram nos CAPS pesquisados, em sistema de rodízio, ou seja, cada encontro grupal foi realizado em um CAPS em duas rodadas. Com base nos objetivos do trabalho e nos eixos temáticos centrais obtidos das entrevistas, a cada encontro foram planejadas atividades utilizando recursos facilitadores para o trabalho grupal, como colagem, construção de cartazes, relaxamento dirigido e técnicas de dinâmica de grupo que serviram como disparadores temáticos (Pichòn-Rivière, 2000a, 2000b) para a reflexão e discussão dos temas.

Como estratégia de coleta de dados na investigação qualitativa, a técnica do Grupo Operativo de Pichón-Riviere (Pichòn-Rivière, 2000a, 2000b) vem sendo utilizada em diversas pesquisas científicas contemporâneas especialmente nas áreas de saúde e educação (Cardoso \& Dall 'Agnol, 2011; Cassol et al, 2012; Van Acker, 2008; Colósio et al, 2007; Grando, 2007; Lucchese \& Barros, 2007; Fortuna, Mishima, Matumoto \& Pereira, 2005). A técnica do Grupo Operativo visa favorecer o protagonismo do grupo na produção de seu referencial conceitual para ser operativo na realidade e aprender, afinando-se assim com os princípios epistemológicos e com os objetivos desta pesquisa.

Os encontros grupais foram coordenados pela pesquisadora e observados por uma psicóloga treinada para desempenhar o papel dentro da técnica do grupo operativo (Jasiner, 2000).

O material de análise consiste na transcrição das entrevistas semiestruturadas e dos encontros grupais com os profissionais, tendo como apoio os registros dos grupos realizados pelo observador silente. Todo o material foi analisado qualitativamente, em momentos distintos, segundo o modelo proposto por Biasoli-Alves e Dias da Silva (1992). As etapas previstas para análise, dentro do modelo proposto, são as seguintes:

- Leitura exaustiva e repetida do material para apreensão de idéias centrais e aspectos relevantes sobre o tema, tendo como pano de fundo os dados complementares;

- Identificação de temas, inicialmente, em cada registro e posteriormente no conjunto do material para a formação de categorias centrais; 
- Sistematização de categorias centrais como fruto de constante movimento entre os dados, a abordagem conceitual e a literatura, até a composição de um quadro significativo de análise;

- Redação final da análise dos dados visando à vinculação com a realidade de modo que os indicativos do trabalho de pesquisa possam embasar reflexões sobre a prática profissional na área e a literatura (Pegoraro \& Caldana, 2008).

Assim, o trabalho de análise dos dados consistiu em uma análise preliminar das entrevistas visando à identificação de eixos temáticos para a realização dos grupos operativos com os profissionais, e posteriormente a construção de categorias de análise ou eixos temáticos, a partir do conjunto de dados obtidos pelas entrevistas e pelos encontros grupais.

Todos os cuidados éticos foram tomados visando atender a Resolução 196/96 do Conselho Nacional de Saúde (conselho.saude.gov/Br/docs/Resoluções/Reso196. doc), em vigor em 2010, ano de realização dada coleta de dados da pesquisa, e a Resolução 16/00 do Conselho Federal de Psicologia (www.pol.org.br/legislacao/doc/ resolucao2000_016.doc).

O projeto foi submetido ao Comitê de Ética em Pesquisa da universidade onde foi realizada a pesquisa e encaminhado à Autarquia de Saúde do município pesquisado, que autorizou a coleta dos dados junto aos profissionais psicólogos dos Centros de Atenção Psicossocial da rede municipal de saúde. Cada participante foi devidamente esclarecido e firmou o Termo de Consentimento Livre e Esclarecido.

\section{RESULTADOS}

Concepções sobre a prática atual: prática clinica tradicional $X$ prática psicossocial $x$ trabalho territorial

A construção das práticas profissionais em psicologia no modelo de atenção psicossocial passa inicialmente pela formação e trajetória profissional dos participantes, que são predominantemente descritas como centradas no modelo clínico tradicional, onde predomina o atendimento em psicoterapia individual e o "setting" clínico. Assim, os participantes relatam um movimento individual de adequação de suas práticas e de sua identidade profissional a formas novas e mais abertas de atendimento, onde se privilegia os atendimentos em grupo e o uso de estratégias diversas, que incluem atividades lúdicas, expressivas, artísticas e esportes, entre outros, além do acompanhamento da pessoa atendida em seu território e o trabalho em rede. A transição de uma prática mais tradicional para aquelas de cunho psicossocial parece acontecer de 
maneira gradual e individual, especialmente no período inicial de trabalho no CAPS, demandando esforços do profissional para o entendimento da proposta e a transformação de suas estratégias de intervenção:

Quando eu entrei aqui, eu me sentia muito estranha. Primeiro porque eu já vim com essa questão de vida clínica e na época tava todo um movimento aqui no CAPS, de tentar desconstruir isso né, porque psicólogo entra no lugar e já tem a ideia de que vai atender assim né, então quando eu entrei foi muito essa coisa, "olha não venha com essa idéia de fazer atendimento clínico aqui", então eu tive que me... procurar. (Márcia - CAPS i)

Ter que lidar com esse psicossocial, eu ainda reluto muito, eu ainda quando eu vejo assim, a minha prática é muito essa coisa mais clínica, né? então eu tenho que ficar me policiando muito, pra ter essa visão mais ampla, não cair novamente numa prática mais clínica aqui. (Amanda - CAPS i)

De modo geral, podemos entender que os profissionais participantes se referem a uma transformação nas concepções tradicionais sobre o papel do psicólogo construídas especialmente no período de formação profissional em psicologia, como psicoterapeuta ou psicólogo clínico, com suas ferramentas de intervenção centradas no modelo clínico tradicional, notadamente a psicoterapia individual e a avaliação psicológica. Tal transformação se inicia a partir do contato com a prática cotidiana, e parece vir carregada de angústias e questionamentos por parte do profissional, que realiza esse percurso individualmente. Nos encontros grupais subsequentes às entrevistas, os participantes aprofundaram o tema da clínica no CAPS, articulando a formação a questionamentos de como pode ser feita uma clínica psicossocial, incluindo a necessidade de avaliar as técnicas utilizadas atualmente:

O que eu tô entendendo dessa conversa é que de verdade mesmo a gente é preparado para a clínica, e eu fico pensando como não se pensar em clínica para o atendimento em saúde mental. Não aquela clínica assim hegemônica, individual, dentro do consultório. Nos serviços de saúde mental os psicólogos muitas vezes vão fazendo e aprendendo por tentativa e erro, e eu acho que falta não só para o psicólogo, porque isso acontece com todos os profissionais, de não ter a formação pro serviço público (Marisa - CAPS III - grupo 6).

Eu acho bem importante pensar, "mas que psicologia?" que técnica é essa? Porque eu não vejo essa preocupação. Incrivelmente, no CAPS Ad a gente consegue trabalhar junto, cada um em uma abordagem teórica diferente, como eu não sei. Muitas vezes eu vejo assim, ta fazendo, mas o quê que ta fazendo? Que técnica é essa que ta aplicando? Isso tem me preocupado muito. (Teresa - CAPS Ad grupo 6)

A fala de Teresa expressa que a abertura na composição das práticas, sem uma reflexão dos profissionais sobre estas, traz a ameaça de haver práticas 
ineficientes, sem objetivos e mesmo sem sentido para quem as executa. Os participantes apontam que, apesar de não haver necessidade de escolha de uma abordagem psicológica única para o CAPS, que a escolha deve ser feita de maneira consciente, com alcances e limites, por cada profissional. Isso coloca o desafio de escolha de estratégias que passa pelas dimensões individuais - que abordagem, com que objetivo - e coletivas: como fazer clínica no CAPS dentro da psicologia sem cair no modelo tradicional?

Eu não acho que todo mundo tem que ser da mesma abordagem, mas eu acho que a gente peca muito quando não sabe em que abordagem está trabalhando, qual é a técnica que tá usando, os objetivos. A gente patina, escorrega com risco de errar feio, né? (Marisa - CAPS III - grupo 6)

É um lugar muito perigoso, porque é muito fácil fazer qualquer coisa, tem que cuidar muito disso (Teresa - CAPS Ad - grupo 6)

As falas acima denotam a preocupação da utilização de um referencial teórico e técnico da psicologia que traga resultados e efetividade para o trabalho. Porém, os participantes não articulam o uso de abordagens psicológicas à clínica ampliada, ou seja, além do uso das abordagens psicológicas, entendemos que seria importante refletir sobre os pressupostos do paradigma psicossocial e a maneira de utilizá-las no contexto da atenção psicossocial.

\section{Práticas inovadoras em saúde mental: a possibilidade de criar}

Outro aspecto relevante em relação às práticas que realizam atualmente é o que os psicólogos participantes trazem como marcas do trabalho que realizam: a possibilidade/necessidade de criar, a tentativa de superação ou diferenciação em relação ao modelo clínico tradicional e a necessidade de reflexão permanente sobre o papel profissional. Tal abertura na composição das práticas é vivenciada de modo muitas vezes contraditório, na medida em que traz o prazer da possibilidade de criar, mas também parece trazer um grande senso de responsabilidade do profissional diante do que cria.

Eu fiquei pensando que o que mantém a gente na saúde mental é o tesão de trabalhar, porque na saúde mental é o único lugar dentro da saúde onde há flexibilidade, onde a gente pode construir. A saúde mental ela tem uma outra, um outro mecanismo de trabalho, né? Que possibilita a práxis, a discussão, né? (Milton - CAPS Ad)

Eu acho que o grande tesão de trabalhar na saúde mental, mas ao mesmo tempo é um desafio, às vezes dá medo, as vezes deixa perdido e tal é a possibilidade infinita de criar, que as vezes nem é possibilidade, é necessidade! aí que dá medo. Saber o que criar. (Marisa - CAPS III - grupo 2) 
O senso de responsabilidade trazido pelos participantes em relação à possibilidade de criação/invenção de práticas parece trazer certa dose de angústia, e pode estar relacionado ao não compartilhamento de idéias, experiências e possibilidades com seus pares ou com a equipe. Na partilha de decisões, idéias e vivências relacionadas às intervenções, partilha-se também a responsabilidade. Nesse ponto, podemos questionar: por que os profissionais, no contexto pesquisado, estão construindo suas práticas predominantemente de maneira individual?

A organização dos processos de trabalho, na forma como é relatada pelos profissionais, não favorece a reflexão e a construção mais coletiva das ações e estratégias profissionais em psicologia, a qual permitiria essa superação de referenciais tradicionais, ou mesmo de uma clínica tradicional no CAPS.

\section{Psicologia e o trabalho territorial}

Em relação ao trabalho efetivamente realizado nos CAPS, os participantes descrevem suas práticas cotidianas e as estratégias de intervenção em psicologia utilizadas, bem como o trabalho técnico de toda a equipe, em duas frentes: as atividades e atendimentos realizados no serviço - triagem, oficinas, grupos terapêuticos e atendimentos individuais com os pacientes e familiares; e outra frente que é o trabalho denominado como técnico de referência de região, onde em cada serviço uma dupla de profissionais de categorias diferentes da equipe constitui uma miniequipe de referência de cada uma das cinco regiões da cidade (norte, sul, leste, oeste e central), da região rural e da população de rua. Dessa maneira, em cada um dos três CAPS da cidade existe uma divisão dos profissionais da equipe em sete miniequipes para atender as regiões da cidade e realizar o trabalho territorial. Segundo os participantes, no trabalho de referência o profissional é responsável por acompanhar os pacientes de sua região no território, fazer contato com a Unidade Básica de Saúde e os outros serviços públicos e comunitários da região destes, realizar visitas domiciliares, realizar grupos com as famílias e no caso do CAPS III, acompanhar o paciente quando fica internado no serviço de hospedagem ou internação breve que o serviço oferece.

O serviço coloca o que faz parte das funções de CAPS, mas tem muito da cara de cada de um, né? Então assim, eu sei que eu tenho que estar na referência de uma região, eu sei que eu tenho que estar na reunião de equipe daquela região, e eu sei que tenho que estar no plantão de acolhimento, eu sei que tenho que ter pelo menos uma 
oficina dirigida, eu sei que eu tenho que ter pelo menos um grupo terapêutico, eu sei que eu tenho que passar visita na hospedagem todos os dias. (Vilma - CAPS III)

Os profissionais descrevem o trabalho territorial realizado por meio desta divisão de miniequipes em cada serviço como a estratégia encontrada para a realização do trabalho territorial, preconizado pelo modelo de atenção em saúde mental vigente e pelo Ministério da Saúde, na medida em que o profissional se desloca até o território do paciente/usuário, seja em visitas domiciliares, seja no contato direto com os recursos comunitários do território, como os serviços de atenção básica em saúde, escolas, serviços de assistência social, entre outros.

$\mathrm{Na}$ verdade a regionalização é uma diretriz no sentido que assim, na medida em que um componente daquela equipe vai pra região ela conhece a dinâmica da região e o que a região pode fazer em prol do paciente, do usuário do serviço, porque se articula com UBS etc, porque a gente trabalha com a questão da reinserção e essa reinserção tem que ser feita ali no território que ele está. (Milton CAPS Ad - grupo 5)

A regionalização do trabalho, ou seja, a divisão da equipe em miniequipes para atender as regiões da cidade, foi a estratégia encontrada para o trabalho territorial no contexto pesquisado. Porém, alguns relatos indicam que os psicólogos não reconhecem o trabalho territorial como prática psicológica.

Eu sou a técnica da região e a psicóloga em mim foi engolida. Quem sou eu mesmo? Tem de bom e de ruim, mas muda, depois que você passa a ser referência de uma região e vê tudo da pessoa, família, se ele come bem ou mal, se toma banho, você tem um acesso a vida da pessoa que jamais você teria num consultório fechado, e eles também se apropriam da gente de uma outra forma. A prática é uma outra coisa. (Vilma, CAPS III - Grupo 4)

Eu acho meio divergente, né? Quando se fala do distanciamento clínico, eu já parei com isso faz tempo, porque no AD a gente se afeta, né? A gente se aproxima muito dos usuários, discute com eles, brinca, conversa sobre futebol, e eu percebo que ir contra essa regra de distanciamento da psicologia na verdade facilita depois o trabalho de reconstrução, de acolhimento, de aproximação e até da escuta do paciente. E por isso escrevi isso aqui: "penso que não sei definir o que carrego da Psicologia em minha prática diária". Tudo se misturou muito, eu particularmente não conseguiria definir o que tem de Psicologia em mim. (Milton - CAPS Ad - Grupo 4)

As falas acima evidenciam a presença de concepções da clínica tradicional e o sentimento de perda da identidade como psicólogo em função deste entrar em contato com aspectos concretos da vida do usuário nas práticas psicossociais desenvolvidas nos serviços e no território. Em outros momentos da discussão no grupo, emergem outras possibilidades de olhar para a prática psicossocial como 
prática psicológica, porem ainda não as identificando como práticas legítimas da psicologia.

\section{DISCUSSÃO}

Ao falarem de suas práticas psicológicas no CAPS, os participantes contrapõem a atuação clínica ao trabalho psicossocial, expressando a concepção de um antagonismo entre as duas propostas. Tal antagonismo presente nas falas sugere que as concepções trazidas pelos profissionais sobre a clínica se referem quase que exclusivamente à clínica tradicional, advinda da formação universitária em psicologia. Tal antagonismo entre a clínica e o psicossocial se torna, dessa maneira, um ponto de angústia para os profissionais psicólogos em relação ao seu papel no serviço, especialmente para os que estão há menos tempo no contexto de CAPS.

No que se refere ao modelo clínico tradicional, Onocko Campos (2001) aponta que, de modo geral, a clínica tradicional é centrada no atendimento individual e na preocupação com a cura ou remoção de sintomas, sejam eles físicos ou psíquicos. Não realiza avaliação de riscos ou ações de prevenção ou promoção de saúde e não considera aspectos sociais ou concretos da vida do sujeito em sua intervenção. No caso da psicologia, a clínica tradicional se configura essencialmente pelo atendimento psicoterapêutico individual e pela avaliação psicológica, predominantemente nas abordagens psicodinâmicas e cognitivo-comportamentais, com a reprodução do setting terapêutico individual no contexto dos serviços públicos de saúde.

Trazendo outro ponto importante, que ecoa com as falas dos participantes da pesquisa, Onocko Campos (2001) aponta que em muitos dos serviços substitutivos de saúde mental a clínica foi negada. Como um dos resultados do processo da reforma psiquiátrica e da luta antimanicomial, no esforço de substituir o modelo asilar e a lógica manicomial, ter-se-ia criado o que a autora chama de focos de cegueira e espaços recalcados, como é o caso da negação da clínica e da doença psiquiátrica. Tal negação teria provocado um esvaziamento da discussão sobre a clínica nos serviços substitutivos de saúde mental, a ideologização dos discursos e, em alguns casos, a idealização da loucura, como também um abismo entre práticas clínicas e psicossociais, por igualar clínica ao conceito de clínica tradicional. Concordamos com a autora quando aponta a necessidade de reflexão sobre a clínica para se avançar na discussão sobre a eficácia do cuidado em saúde mental, considerando a existência de outras possibilidades de clínica para além da tradicional. Nesta direção, não se trata de negar ou abolir a clínica para se construir o modo psicossocial de atenção em saúde mental, mas se torna de fundamental importância a ampliação do debate 
sobre a clínica possível no serviço público de saúde mental, que esteja em sintonia com os pressupostos do paradigma psicossocial.

Ferreira Neto (2008) aponta que as concepções e tendências em desenvolvimento no campo da clínica em psicologia necessitam de revisão em duas direções: a inclusão do social como fator fundamental para investigar a constituição da subjetividade e para a intervenção, e o segundo é a consideração do campo multidisciplinar e transdisciplinar como base indispensável para a produção do conhecimento. Dessa maneira, segundo o autor, decreta-se o fim do solipsismo para se pensar tanto a subjetividade quanto o conhecimento.

Segundo Passos (2007), essa mudança paradigmática deve ser compreendida em sua essência no que diz respeito à atuação clínica, para que na prática não ocorram "pseudo" mudanças, como por exemplo, a clínica tradicional acontecendo nos serviços públicos, alterando apenas o lugar e a clientela atendida. Para além da reprodução de modelos antigos em novos contextos ou da negação total da clínica, é preciso alterar a concepção que se tem do sujeito e do uso de métodos e técnicas voltados para a "normalização" da subjetividade.

Surge assim a proposta da clínica ampliada e compartilhada (Campos \& Amaral, 2007). Ampliada porque amplia o "objeto de trabalho" da clínica, agregando a ele, além das doenças, também problemas de saúde - situações que ampliam o risco ou vulnerabilidade das pessoas.

É importante destacar que, como aponta Cunha (2010), todo o esforço de síntese de saberes não vinculados ao modelo biomédico e no caso da psicologia, o modelo clínico tradicional, bem como a incorporação de críticas a eles não significam, de forma alguma, abrir mão de recursos de intervenção dos modelos tradicionais, mas constatar que o conhecimento biomédico, tradicional e hegemônico na clínica de diversos profissionais é comprovadamente insuficiente e potencialmente danoso quando utilizado de forma excludente. Nesta direção, a medicação, os procedimentos e saberes biomédicos e do modelo clínico tradicional em psicologia constituem uma das forças para o cuidado do sujeito, mas não a única ou principal.

Segundo Campos e Amaral (2007), a ampliação mais importante da clínica tradicional para a clínica ampliada seria a consideração de que os problemas de saúde ou doença estão sempre "encarnados" em sujeitos, em pessoas. E essas pessoas possuem condições concretas de existência, que devem ser consideradas no cuidado em saúde Assim, o autor propõe que a principal ampliação seja a construção da "clínica do sujeito".

Na mesma direção, o autor considera essencial a ampliação do objetivo ou da finalidade do trabalho clínico: além de buscar a produção de saúde, por distintos meios - curativos, preventivos, de reabilitação ou com cuidados paliativos -, a clínica poderá também contribuir para a ampliação do grau de autonomia dos usuários, ou seja, para a ampliação da capacidade do usuário de lidar com sua própria rede ou sistema de dependências. Aqui, o contexto social 
e cultural, e, até mesmo, a própria subjetividade e a relação de afetos inevitavelmente estarão envolvidos.

A ampliação do grau de autonomia pode ser avaliada pelo aumento da capacidade dos usuários compreenderem e atuarem sobre si mesmos e sobre o mundo da vida. Campos e Amaral (2007) apontam que o grau de autonomia se mede pela capacidade de autocuidado, de compreensão sobre o processo saúde/enfermidade, pela capacidade de usar o poder e de estabelecer compromisso e contrato com outros.

Torna-se evidente que a proposta da clínica ampliada se afina com os pressupostos da reforma psiquiátrica e do modelo de atenção psicossocial, trazendo novas possibilidades para a clínica que ampliam o olhar e a atuação para além do fazer biomédico e, no caso da psicologia, para além da clínica tradicional.

Nesse sentido, a clínica ampliada traz também aproximações em relação à "clínica peripatética" de Lancetti (2009), que propõe que a clínica é muito mais do que o consultório, ou setting privado de trabalho clinico. Lancetti propõe experiências clínicas em movimento, nos espaços abertos da vida social.

Concordamos com Lancetti (2009) e com Cambuy e Amatuzzi (2012) ao apontarem que "fazer clínica" na psicologia não significa apenas lidar com a interioridade psicológica do sujeito, mas também com toda a rede de subjetividade que o envolve. Faz-se necessário e urgente criar novas modalidades de intervenção que produzam e provoquem reações nos indivíduos para que eles passem a ordenar de modo mais criativo suas práticas sociais e psíquicas. Assim como nós, os autores supracitados apostam na possibilidade da construção de novos paradigmas tanto para a prática como para a formação do psicólogo clínico, como também na potência terapêutica de espaços diferentes daqueles da psicoterapia convencional.

Os participantes de nossa pesquisa evidenciam tal potência e a realização de novas possibilidades da clínica no serviço de saúde mental. Faz-se necessário "nomear" e se apropriar de tais experiências como práticas legítimas da psicologia.

\section{CONSIDERAÇÕES FINAIS}

No ano de 2013, o Conselho Federal de Psicologia, por meio do Centro de Referência Técnica em Psicologia e Políticas Públicas - CREPOP, lançou o documento "Referências Técnicas para Atuação de Psicóloga (os) no CAPS Centro de Atenção Psicossocial" (Conselho Federal de Psicologia, 2013). O documento apresenta os resultados e a discussão dos dados de uma pesquisa realizada com psicólogos trabalhadores de CAPS de todo o país.

Em termos de produção de conhecimento na área, entendemos que a grande proximidade com os resultados e temas da pesquisa realizada pelo 
Conselho Federal de Psicologia (Conselho Federal de Psicologia, 2013), em época semelhante a esta pesquisa, sobre a atuação do psicólogo nos CAPS do Brasil, é uma evidência bastante positiva, que corrobora as ideias centrais deste trabalho.

Em ambas as pesquisas constata-se que a clínica dos CAPS distancia-se do fazer clínico tradicional, visando promover uma elaboração subjetiva e reabilitação no processo de construção da autonomia e da capacidade de cada usuário. Por isso, agrega à psicoterapia e ao medicamento, a potência de outros recursos e intervenções. Não nega os recursos tradicionais, mas inclui outras estratégias como oficinas, assembleias, permanência, hospitalidade, mediação das relações entre os sujeitos e seus familiares. Nessa clínica ampliada no CAPS, as referências e as redes dos sujeitos têm tanto valor quanto os recursos da ciência e da técnica. Aqui, o cuidado passa pela construção de condições de liberdade e capacidade de se inserir na cidade, de fazer caber a diversidade na singularidade dos sujeitos, no universal da cidadania e do protagonismo social. (Conselho Federal de Psicologia, 2013).

$\mathrm{Na}$ presente pesquisa entendemos, a partir dos relatos dos próprios psicólogos, que estes realizam ações que seriam consideradas de clínica ampliada nos CAPS, como as oficinas, as visitas, o trabalho no território, o trabalho em rede, além do trabalho psicoterapêutico realizado com os usuários no serviço. Porém, no momento da realização da pesquisa, tais ações ficaram um tanto "sem nome" e muitas vezes não identificadas como ações do psicólogo, indicando que a identidade profissional ainda está calcada, em grande medida, no papel tradicional.

É importante mencionar que a clínica ampliada é uma estratégia incorporada como política pública no Brasil, sendo parte integrante da Política Nacional de Humanização do SUS (Ministério da Saúde, 2004, 2009). Nessa proposta, os instrumentos Clínica Ampliada, Equipes de Referência e Projetos Terapêuticos Singulares têm-se mostrado como dispositivos resolutivos, tanto no âmbito da atenção quanto no âmbito da gestão de serviços e redes de saúde, o que inclui a saúde mental. Porém, como mostram os dados dessa pesquisa, as inovações científicas, paradigmáticas, técnicas e mesmo as políticas públicas orientadoras para a prática psicossocial, parecem ainda ter pouca penetração na "ponta", ou seja, na atuação cotidiana dos profissionais dos serviços de saúde mental. Precisam, dessa maneira, ser discutidas e refletidas nos processos de formação profissional e educação permanente na área da psicologia e dos profissionais de saúde, ou seja, ser cada vez mais parte integrante dos currículos universitários e das estratégias de educação permanente, oferecendo essas novas perspectivas epistemológicas, paradigmáticas que se traduzam em estratégias de intervenção para os profissionais em formação e também para aqueles que estão inseridos nos serviços da rede de atenção psicossocial e têm cotidianamente a tarefa de construir e efetivar o paradigma psicossocial nas práticas de cada dia. 


\section{REFERÊNCIAS}

Amarante, P. (2011). Saúde mental e atenção psicossocial. Rio de Janeiro: Editora Fiocruz.

Biasoli-Alves, Z.M.M. \& Dias-da-Silva, M. G. F. (1992). Análise qualitativa de dados de entrevista: uma proposta. Paidéia (Ribeirão Preto), 2, 61-69.

Bogdan, R. \& Biklen, S. (1994). Investigação Qualitativa em Educação. Portugal: Porto Editora.

Cambuy, K. \& Amatuzzi, M. M. (2012). Experiências comunitárias: repensando a clínica psicológica no SUS. Psicologia e Sociedade, 24(3), 574-683.

Campos, G.W.S. \& Amaral, M.A. (2007). A clínica ampliada e compartilhada, a gestão democrática e redes de atenção como referenciais teóricooperacionais para a reforma do hospital. Ciência e Saúde Coletiva, 12(4), 849-859.

Cardoso, A. S. F. \& Dall'Agnol, C.M.(2011). Processo grupal: reflexões de uma equipe de enfermagem. Revista da escola de enfermagem da USP, 45(6), 1412-1418.

Cassol, P. B., Terra, M.G, Mostardeiro, S.C.T.S., Gonçalves, M.O. \& Pinheiro, U.M.S. (2012). Tratamento em um grupo operativo em saúde: percepção dos usuários de álcool e outras drogas. Revista Gaúcha de Enfermagem, 33(1), 132-138.

Colosio, R., Fernandes, M. I. A., Bergamaschi, D.P., Scarcelli, I. R., Lopes, I. C. \& Hearst, N. et al. (2007). Prevenção de infecção pelo HIV por intermédio da utilização do grupo operativo entre homens que fazem sexo com homens. Cadernos de Saúde Pública (FIOCRUZ), 23, 949-959.

Conselho Federal de Psicologia - CFP. (2005). Pesquisa de Opinião WHO - Quem é o psicólogo brasileiro - Ano 2001, Brasília: Autor. Recuperado de: http://site.cfp.org.br/wp-content/uploads/2005/05/Pesquisa_WHO.pdf

Conselho Federal de Psicologia - CFP. (2013). Referências técnicas para atuação de Psicólogas (os) no CAPS - Centro de Atenção Psicossocial. Brasília: Autor. Recuperado de: http://crepop.pol.org.br/novo/wpcontent/uploads/2013/07/MIOLO_TECNICAS_DE_ATUACAO2.pdf

Costa- Rosa, A. (2000). O modo Psicossocial: um paradigma das práticas substitutivas ao modo asilar. In: Amarante, P. (org.) Ensaios: subjetividade, saúde mental e sociedade. Rio de Janeiro: Editora Fiocruz.

Cunha, G. T. (2010). A Construção da Clínica Ampliada na Atenção Básica. São Paulo: Hucitec.

Dimenstein, M. (2000). A cultura profissional do psicólogo e o ideário individualista: implicações para a prática no campo da assistência pública à saúde. Estudos de Psicologia, 5(1), 95-121. 
Dimenstein, M. (2004). A reorientação da atenção em saúde mental: sobre a qualidade e humanização da assistência. Psicologia: ciência e profissão, 24(4), 112-117.

Dimenstein, M. (2006). A prática dos psicólogos no Sistema Único de Saúde/SUS. In: Conselho Federal de Psicologia. Cartilha do I Fórum Nacional de Psicologia e Saúde Pública: contribuições técnicas e políticas para avançar o SUS (pp.08-16). Brasília: Autor.

Dimenstein, M. \& Macedo, J. P. (2012). Formação em Psicologia: requisitos para atuação na atenção primária e psicossocial. Psicologia: Ciência e Profissão, 32(n.spe), 232-245.

Ferreira Neto, J. L. (2008). Intervenção psicossocial em saúde e formação do psicólogo. Psicologia e Sociedade, 20(1), 62-69.

Figueiredo, V.V. \& Rodrigues, M.M.P. (2004). Atuação do psicólogo nos CAPS do Estado do Espírito Santo. Psicologia em Estudo, 9(2), 173-181.

Fortuna, C.M., Mishima, S.M., Matumoto, S. \& Pereira, M.J.B. (2005). O trabalho de equipe no programa de saúde da família: reflexões a partir de conceitos do processo grupal e de grupos operativos. Revista Latino-Americana de Enfermagem, 13(2), 262-268.

Grando, M.K. (2007). Reuniões de equipe na Estratégia Saúde da Família a partir do referencial pichoniano de grupo operativo. Dissertação de Mestrado, Escola de Enfermagem, Universidade Federal do Rio Grande do Sul. Porto Alegre.

Jasiner, C. (2000). Observando la observación: adiós a Pilatos. Revista Campo Grupal, Buenos Aires, 18, 17-25.

Lancetti, A. (2009). Clínica Peripatética. São Paulo: Hucitec.

Lucchese, R. \& Barros, S. (2007). A utilização do grupo operativo como método de coleta de dados em pesquisa qualitativa. Revista Eletrônica de Enfermagem, 9(3), 796-805.

Mezzina, R. (2005). Paradigm Shift in Psychiatry: Processes and Outcomes. In: Ramon, S. \& Williams, J.E. Mental Health at the Crossroads: The Promise of the Psychosocial Approach (pp.81-93). London: Ashgate.

Ministério da Saúde, (2004a). Secretaria de Atenção à Saúde. Núcleo Técnico da Política Nacional de Humanização. Clínica ampliada, equipe de referência e projeto terapêutico singular / Ministério da Saúde, Secretaria de Atenção à Saúde, Núcleo Técnico da Política Nacional de Humanização - 1. ed. Brasília: Autor.

Ministério da Saúde. (2004b). Legislação em Saúde Mental - 1990/2004. Brasília: Autor.

Ministério da Saúde. (2005). Secretaria de Atenção à Saúde. DAPE. Coordenação Geral de Saúde Mental. Reforma psiquiátrica e política de saúde mental no Brasil. Brasília: Autor. Recuperado de http://bvsms.saude.gov.br/bvs/publicacoes/Relatorio15_anos_Caracas.pdf 
Ministério da Saúde. (2007). Secretaria de Atenção à Saúde/DAPE. Saúde Mental no SUS: acesso ao tratamento e mudança do modelo de atenção. Relatório de Gestão 2003-2006. Brasília: Autor. Recuperado de: http://bvsms.saude.gov.br/bvs/publicacoes/relatorio_gestao_saude_mental _2003-2006.pdf

Ministério da Saúde. (2009). Secretaria de Atenção à Saúde. Política Nacional de Humanização da Atenção e Gestão do SUS. Clínica ampliada e compartilhada. Brasília: Autor. Recuperado de: <http://bvsms.saude.gov.br/bvs/publicacoes/clinica_ampliada_compartilha da.pdf>

Ministério da Saúde. (2011). Portaria GM/MS no 3.088/2011 - preconiza o atendimento a pessoas com sofrimento ou transtorno mental e com necessidades decorrentes do uso de crack, álcool e outras drogas. Brasília: Autor.

Onocko Campos, R. T. (2001). Clínica: a palavra negada - sobre as práticas clínicas nos serviços substitutivos de Saúde Mental. Saúde em Debate, 25(58), 98-111.

Pegoraro, R.F. \& Caldana, R.H.L. (2008). Sofrimento psíquico em familiares de usuários de um Centro de Atenção Psicossocial. Interface - Comunicação, Saúde, Educação, 12(25), 295-307.

Passos, E. (2007). Ética e Psicologia: teoria e prática. São Paulo: Vetor.

Pereira, F. M. \& Pereira Neto, A. (2003). O psicólogo no Brasil: notas sobre seu processo de profissionalização. Psicologia em Estudo, 8(2) 19-27.

Pichón-Rivière, E. (2000a). O processo grupal. São Paulo: Martins Fontes (Trabalho original publicado em 1983).

Pichón-Rivière, E. (2000b). Teoria do vínculo. São Paulo: Martins Fontes (Trabalho original publicado em 1982).

Silva, R.C. (1998). A falsa dicotomia qualitativo - quantitativo: Paradigmas que informam nossas práticas de pesquisas. In: Romanelli, G. (org.) Diálogos metodológicos sobre prática de pesquisa ( $p p$. 159-174). Ribeirão Preto: Legis Summa.

Spink, M.J. (2003). Psicologia social e saúde - prática, saberes e sentidos. Petrópolis: Vozes.

Van Acker, M. T. V. (2008). A reflexão e a prática docente: considerações a partir de uma pesquisa-ação. Tese de Doutorado, Faculdade de Educação, Universidade de São Paulo, São Paulo.

Yamamoto, O.H. (2012). 50 anos de profissão: responsabilidade social ou projeto ético-político? Psicologia: ciência e profissão, 32(n.spe), 6-17.

Yasui, S. \& Costa-Rosa, A. (2008). A Estratégia Atenção Psicossocial: desafio na prática dos novos dispositivos de Saúde Mental. Saúde em Debate, $32(78 / 79 / 80), 27-37$. 
Yasui, S. (2010). Rupturas e encontros: desafios da reforma psiquiátrica brasileira. Rio de Janeiro: Fiocruz.

Sobre as autoras:

Thaís Thomé Seni da Silva e Oliveira é Psicóloga, Mestre, Doutora e Pós-doutora em Psicologia pela Universidade de São Paulo. Atualmente é pesquisadora colaboradora do Departamento de Saúde Mental de Trieste - Centro Colaborador da OMS na área de Saúde Mental. thasenne@gmail.com

Regina Helena Lima Caldana é Psicóloga, Mestre e Doutora pela Universidade Federal de São Carlos e docente do curso de Psicologia da FFCLRP - Universidade de São Paulo. rhlcalda@ffclrp.usp.br

Recebido em: 03/02/2016

Revisado em: 25/03/2016

Aceito em: 06/04/2016 